\title{
Tamoxifen-loaded nanostructured lipid carrier as a drug delivery system: characterization, stability assessment and cytotoxicity
}

\begin{abstract}
Cancer nanotherapeutics is beginning to overwhelm the global research and viewed to be the revolutionary treatment regime in the medical field. This investigation describes the development of a stable nanostructured lipid carrier (NLC) system as carrier for Tamoxifen (TAM). The TAM-loaded NLC (TAM-NLC) developed with $200 \mathrm{mg}$ of TAM showed a spherical particle with the size of $46.6 \mathrm{~nm}$, polydispersity index of 0.267 , entrapment efficiency of $99.74 \%$ and with the zeta potential of $-23.78 \mathrm{mV}$. Besides, the equivalent cytotoxicity of TAM and TAM-NLC to human (MCF-7) and mice (4T1) mammary breast cancer cell lines were observed. Incubating the formulation at the physiological $\mathrm{pH}$ resulted into reduced Ostwald ripening rate but without any significant change in the absorptivity. When coupled with the measurements of zeta potential and Ostwald ripening rate, the absorbance assay may be used to predict the long-term stability of drug-loaded nanoparticle formulations. The results of the study also suggest that TAM-NLC is a promising drug delivery system for breast cancer therapy. This is the first encouraging report on the in vitro effect of TAM-NLC against human and mouse mammary adenocarcinoma cell lines.
\end{abstract}

Keyword: Lipid nanoparticles; Tamoxifen; Absorbance; Ostwald ripening; Mammary adenocarcinoma cell line; Drug delivery; Nanostructured lipid carrrier. 